\title{
Strength of thin-walled elastic building structures
}

\author{
Lilya Kharasova*1[0000-0002-4713-3930] \\ ${ }^{1}$ Kazan Federal University - Naberezhnye Chelny Institute, 423812 Naberezhnye Chelny, Russia
}

\begin{abstract}
The existence theorem is proved within the framework of the shear model by S.P. Timoshenko. The stress-strain state of elastic inhomogeneous isotropic shallow thin-walled shell constructions is studied. The stress-strain state of shell constructions is described by a system of the five equilibrium equations and by the five static boundary conditions with respect to generalized displacements. The aim of the work is to find generalized displacements from a system of equilibrium equations that satisfy given static boundary conditions. The research is based on integral representations for generalized displacements containing arbitrary holomorphic functions. Holomorphic functions are found so that the generalized displacements should satisfy five static boundary conditions. The integral representations constructed this way allow to obtain a nonlinear operator equation. The solvability of the nonlinear equation is established with the use of contraction mappings principle.
\end{abstract}

Keywords. Building constructions of the shell type, stress-strain state, equilibrium equations, static boundary conditions, generalized displacements.

\section{Introduction}

The existence of existence theorems makes it easy to prove the convergence of numerical methods to an exact real solution and contributes to a deep understanding of the studied mechanical phenomena. Therefore, a rigorous study of boundary value problem solvability, the proof of existence theorems and the development of the methods for finding solutions are very urgent problems in the mathematical theory of elasticity. Currently, there is a large number of works devoted to the strength of thin-walled shell structures, taking into account the geometric and/or physical nonlinearity [1-9]. This is due to the widespread use of elastic thin-walled shell structures in aviation, space technology, shipbuilding, mechanical engineering and construction. In very rare cases, nonlinear problems are solved in a closed form. For this reason, a wide range of approximate methods with the use of computers is used to solve them. When solving problems numerically, the problem of convergence of the numerical solution to the exact (real) solution of the problem always comes to the fore. The solution to this problem, as is known, is based on a rigorous mathematical study of the stressstrain state of thin-walled shell structures. At present, this problem is sufficiently fully studied in the framework of the simplest Kirchhoff-Love model [10-17]. Questions related to the qualitative study of the stress-strain state within the framework of more general models of the theory of thin-walled shell structures that do not rely on the Kirchhoff-Love hypotheses

*Corresponding author: kharasova.liya@mail.ru 
were included in the well-known list of unsolved problems of the mathematical theory of shells by I.I. Vorovich [10] and remained open until recently. To date, there is a number of works [18-24] devoted to the study of the stress-strain state in the framework of the Timoshenko shear model. The research in [18-24] is based on integral representations for generalized displacements containing arbitrary holomorphic functions that are found in such a way that the generalized displacements satisfy the given boundary conditions. Two approaches are used to build them. The first approach is based on the application of explicit representations of solutions to Riemann - Hilbert problems for holomorphic functions in the unit circle. Therefore, a flat region homeomorphic to the median surface of the shell is either assumed to be a unit circle from the very beginning [18-20], or conformally mapped to a unit circle [22], [24]. In the second approach, the theory of one-dimensional singular integral equations is used to determine holomorphic functions [21], [23]. In this paper, the conformal mapping method is used to study a nonlinear problem for arbitrary flat shells under different boundary conditions.

\section{Materials and methods}

In this paper, we consider the following model of the theory of shallow shells by S.P. Timoshenko:

1) the relationship of deformation-displacement [25, pp. 168-170, 269]:

$$
\begin{gathered}
\gamma_{j j}^{0}=w_{j \alpha^{j}}-k_{j} w_{3}+w_{3 \alpha^{j}}^{2} / 2, j=1,2, \gamma_{12}^{0}=w_{1 \alpha^{2}}+w_{2 \alpha^{1}}+w_{3 \alpha^{1}} w_{3 \alpha^{2}}, \\
\gamma_{j j}^{1}=\psi_{j \alpha^{j}}, j=1,2, \gamma_{12}^{1}=\psi_{1 \alpha^{2}}+\psi_{2 \alpha^{1}}, \\
\gamma_{j 3}^{0}=w_{3 \alpha^{j}}+\psi_{j}, j=1,2, \gamma_{33}^{0}=\gamma_{k 3}^{0} \equiv 0, k=\overline{1,3}
\end{gathered}
$$

where $\gamma_{i j}^{k}(i, j=\overline{1,3}, k=0,1)$ - the components of the deformations of the shell middle surface $S_{0} ; w_{j}(j=1.2)$ and $w_{3}$ - tangential and normal displacements of points $S_{0} ; \Psi_{j}(j=1.2)$ - the angles of normal section rotation; $\alpha^{I}, \alpha^{2}$ - Cartesian coordinates of points of a flat bounded domain $\Omega$ with the boundary $\Gamma$, homeomorphic $S_{0}$;

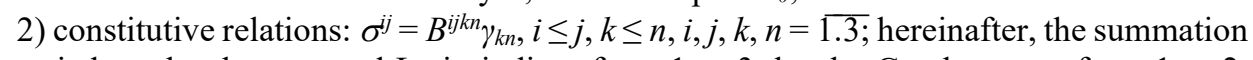
is carried out by the repeated Latin indices from 1 to 3 , by the Greek ones - from 1 to 2 , where $\gamma_{k n}=\gamma_{k n}^{0}+\alpha^{3} \gamma_{j k n}^{1} ; B^{i j k n}-$ the elastic characteristics of the shell: $B^{1111}=B^{2222}=E /\left(1-\mu^{2}\right)$, $B^{1122}=\mu E /\left(1-\mu^{2}\right), B^{1212}=E /(2(1+\mu)), B^{1313}=B^{2323}=E k^{2} /(2(1+\mu))$ the rest $B^{i j k n}=0 ; \mu=$ const - Poisson's ratio, $E=$ const - Young's modulus, $k_{1}, k_{2}=$ const - the main curvatures; $k^{2}=$ const - shear coefficient;

3) boundary conditions on $\Gamma$ :

$$
w_{l}=\Psi_{l}=0
$$

4) mass $\vec{F}\left(\alpha^{1}, \alpha^{2}, \alpha^{3}\right)$ and surface $\overrightarrow{F^{ \pm}}\left(\alpha^{1}, \alpha^{2}\right)$ forces act on the shell, and the forces $\overrightarrow{F^{0}}\left(s, \alpha^{3}\right)$ are applied at the shell boundary.

Using the variational Lagrange principle, we obtain the equilibrium equations:

$$
\begin{gathered}
T_{\alpha^{\lambda}}^{i \lambda}+R^{i}=0, \quad i=1,2, \\
T_{\alpha^{\lambda}}^{\lambda 3}+k_{\lambda} T^{\lambda \mu}+\left(T^{\lambda \mu} w_{3 \alpha^{\lambda}}\right)_{\alpha^{\mu}}+R^{3}=0, \\
M_{\alpha^{\lambda}}^{i \lambda}-T^{i 3}+L^{i}=0, \quad i=1,2
\end{gathered}
$$

and the static boundary conditions on $\Gamma$ :

$$
\begin{gathered}
T^{12} d \alpha^{2} / d s-T^{22} d \alpha^{1} / d s=P^{2}(s) \\
T^{13} d \alpha^{2} / d s-T^{23} d \alpha^{1} / d s+T^{11} w_{3 \alpha^{1}} d \alpha^{2} / d s-T^{22} w_{3 \alpha^{2}} d \alpha^{1} / d s+ \\
+T^{12}\left(w_{3 \alpha^{2}} d \alpha^{2} / d s-w_{3 \alpha^{1}} d \alpha^{1} / d s\right)=P^{3}(s),
\end{gathered}
$$




$$
M^{12} d \alpha^{2} / d s-M^{22} d \alpha^{1} / d s=N^{2}(s)
$$

where $T^{i j}$ - the efforts, $M^{i j}$ - the moments:

$$
T^{i j} \equiv T^{i j}(a)=D_{0}^{i j k n} \gamma_{k n}^{0}, M^{i j} \equiv M^{i j}(a)=D_{2}^{i j k n} \gamma_{k n}^{1}, D i j k n_{m}=\int_{-h / 2}^{h / 2} B^{i j k n}\left(\alpha^{3}\right)^{m} d \alpha^{3} ;
$$

$R^{i}(i=\overline{1.3}), L^{j}(j=1.2), P^{2}, P^{3}, N^{2}$ depend on external forces; $a=\left(w_{1}, w_{2}, w_{3}, \Psi_{l}, \Psi_{2}\right)-$ the vector of generalized displacements, $h=$ const - shell thickness.

\subsection{Problem A}

It is required to find a solution to the system (3) that satisfies the boundary conditions (2), (4).

We will study the boundary value problem A in a generalized setting. Let the following conditions be satisfied:

a) $\Omega$ is a simply connected domain with the boundary $\Gamma \in C_{\beta}{ }^{l}$;

b) $\vec{F} \in L_{p}(\Omega) \times L_{1}[-h / 2 ; h / 2], \overrightarrow{F^{ \pm}} \in L_{p}(\Omega), \vec{F}^{0} \in C_{\beta}(\Gamma) \times L_{1}[-h / 2 ; h / 2]$; here and below, everywhere $p>2.0<\beta<1$.

During displacements, the equilibrium equations (3) take the following form:

$$
\begin{gathered}
w_{1 \alpha^{1} \alpha^{1}}+\mu_{1} w_{1 \alpha^{2} \alpha^{2}}+\mu_{2} w_{2 \alpha^{1} \alpha^{2}}=f_{1}, \\
\mu_{1} w_{2 \alpha^{1} \alpha^{1}}+w_{2 \alpha^{2} \alpha^{2}}+\mu_{2} w_{1 \alpha^{1} \alpha^{2}}=f_{2}, \\
k^{2} \mu_{1}\left(w_{3 \alpha^{1} \alpha^{1}}+w_{3 \alpha^{2} \alpha^{2}}+\psi_{1 \alpha^{1}}+\psi_{2 \alpha^{2}}\right)+k_{3} w_{1 \alpha^{1}}+k_{4} w_{2 \alpha^{2}}-k_{5} w_{3}+ \\
+k_{3} w_{3 \alpha^{1}}^{2} / 2+k_{4} w_{3 \alpha^{2}}^{2} / 2+\beta_{2}\left[\left(T^{\lambda \mu} w_{3 \alpha^{2}}\right)_{\alpha^{\mu}}+R^{3}\right]=0, \\
\psi_{1 \alpha^{1} \alpha^{1}}+\mu_{1} \psi_{1 \alpha^{2} \alpha^{2}}+\mu_{2} \psi_{2 \alpha^{1} \alpha^{2}}=\widetilde{g}_{1}, \\
\mu_{1} \psi_{2 \alpha^{1} \alpha^{1}}+\psi_{2 \alpha^{2} \alpha^{2}}+\mu_{2} \psi_{1 \alpha^{1} \alpha^{2}}=\widetilde{g}_{2},
\end{gathered}
$$

the boundary conditions on $\Gamma$ are transformed to the following form:

$$
\begin{gathered}
\mu_{1}\left(w_{1 \alpha^{2}}+w_{2 \alpha^{1}}\right)(t) d \alpha^{2} / d s-\left(\mu w_{1 \alpha^{1}}+w_{2 \alpha^{2}}\right)(t) d \alpha^{1} / d s=\phi_{2}\left(w_{3}\right)(t) \\
\mu_{1}\left(\psi_{1 \alpha^{2}}+\psi_{2 \alpha^{1}}\right)(t) d \alpha^{2} / d s-\left(\mu \psi_{1 \alpha^{1}}+\psi_{2 \alpha^{2}}\right)(t) d \alpha^{1} / d s=\tilde{\phi}_{2}(t) \\
T^{13} d \alpha^{2} / d s-T^{23} d \alpha^{1} / d s+T^{11} w_{3 \alpha^{1}} d \alpha^{2} / d s-T^{22} w_{3 \alpha^{2}} d \alpha^{1} / d s+ \\
+T^{12}\left(w_{3 \alpha^{2}} d \alpha^{2} / d s-w_{3 \alpha^{1}} d \alpha^{1} / d s\right)=P^{3}(s) .
\end{gathered}
$$

The following designations are adopted in (6)-(9):

$$
\begin{gathered}
f_{1} \equiv f_{1}\left(w_{3}\right)=k_{3} w_{3 \alpha^{1}}-w_{3 \alpha^{1}} w_{3 \alpha^{1} \alpha^{1}}-\mu_{2} w_{3 \alpha^{2}} w_{3 \alpha^{1} \alpha^{2}}-\mu_{1} w_{3 \alpha^{1}} w_{3 \alpha^{2} \alpha^{2}}-\beta_{2} R^{1}, \\
f_{2} \equiv f_{2}\left(w_{3}\right)=k_{4} w_{3 \alpha^{2}}-w_{3 \alpha^{2}} w_{3 \alpha^{2} \alpha^{2}}-\mu_{2} w_{3 \alpha^{1}} w_{3 \alpha^{1} \alpha^{2}}-\mu_{1} w_{3 \alpha^{2}} w_{3 \alpha^{1} \alpha^{1}}-\beta_{2} R^{2}, \\
\tilde{g}_{j}=g_{j}+k_{0} \psi_{j}, g_{j} \equiv g_{j}\left(w_{3}\right)=k_{0} w_{3 \alpha^{j}}-\beta_{1} L^{j}, \mu_{1}=(1-\mu) / 2, \mu_{2}=(1+\mu) / 2, \\
\varphi_{2}\left(w_{3}\right)(t)=\beta_{2} P^{2}(s)+\left[-k_{4} w_{3}(t)+\mu w_{3 \alpha^{1}}^{2}(t) / 2+w_{3 \alpha^{2}}^{2}(t) / 2\right] d \alpha^{1} / d s- \\
-\mu_{1} w_{3 \alpha^{1}}(t) w_{3 \alpha^{2}}(t) d \alpha^{2} / d s, \widetilde{\varphi}_{2}(t)=\beta_{1} N^{2}(s), \\
t=t(s)=\alpha^{1}(s)+i \alpha^{2}(s), k_{3}=k_{1}+\mu k_{2}, k_{4}=k_{2}+\mu k_{1}, k_{5}=k_{1}^{2}+k_{2}^{2}+2 \mu k_{1} k_{2}, \\
k_{0}=6 k^{2}(1-\mu) / h^{2}, \beta_{1}=12\left(1-\mu^{2}\right) /\left(h^{3} E\right), \beta_{2}=\left(1-\mu^{2}\right) /(E h) .
\end{gathered}
$$

\subsection{Definition}

A generalized solution of the problem A is the vector of generalized displacements $a=\left(w_{l}\right.$, $\left.w_{2}, w_{3}, \Psi_{l}, \Psi_{2}\right) \in W_{p}^{(2)}(\Omega), p>2$, almost everywhere satisfying the system (6) and pointwise boundary conditions (2), (7)-(9). 
Let's consider the system of the first two equations in (6), in which the deflection is assumed to be fixed temporarily. The general solution of the system (1) has the form [18]:

$$
\omega_{0}(z)=w_{2}+i w_{1}=\Phi_{2}(z)+i T d\left[\Phi_{1}+T f\right](z), z=\alpha^{1}+i \alpha^{2}, f=\left(f_{1}+i f_{2}\right) / 2,
$$

where $\Phi_{1}(z) \in C_{\alpha}(\bar{\Omega}), \Phi_{2}(z) \in C_{\alpha}^{1}(\bar{\Omega})$ - arbitrary holomorphic functions;

$$
T f=-\frac{1}{\pi} \iint_{\Omega} \frac{f(\zeta)}{\zeta-z} d \xi d \eta, \zeta=\xi+i \eta, d[g]=d_{1} g+d_{2} \bar{g}, d_{j}=\left(\mu_{1}+(-1)^{j}\right) /\left(4 \mu_{1}\right), j=1,2 .
$$

Let us find the holomorphic functions $\Phi_{j}(z), j=1.2$ so that the tangential displacements $w_{1}, w_{2}$ (11) satisfy the boundary conditions (2), (7). Following [22], for tangential displacements $w_{1}, w_{2}$ under the condition of solvability of the form:

we obtain the required representation:

$$
\int_{\Gamma} P^{2}(s) d s+\iint_{\Omega} R^{2} d \alpha^{1} d \alpha^{2}=0,
$$

where

$$
\begin{gathered}
\omega_{0}(z)=H_{0} w_{3}(z)+c_{0}, z \in \Omega \\
H_{0} w_{3}(z)=\Phi_{2}\left[l\left(w_{3}\right)\right](\Psi(z))+i T d\left[\Phi_{1}\left[l\left(w_{3}\right)\right](\Psi(\zeta))+T f\left(w_{3}\right)(\zeta)\right](z),
\end{gathered}
$$

$$
\begin{gathered}
\Phi_{2}\left[l\left(w_{3}\right)\right](\psi(z))=-\frac{1}{2 \pi} \int_{\partial K}\left(\operatorname{Re} T d\left[\Phi_{1}\left[l\left(w_{3}\right)\right]\right](t)+\operatorname{Re} T d[T f](t)\right) \frac{t+\psi(z)}{t-\psi(z)} \frac{d t}{t}, \\
\Phi_{1}\left[l\left(w_{3}\right)\right](z)=2(\mu-1) S_{\partial K}(\operatorname{Re} T d[T f](\varphi(\tau)))(\widetilde{\zeta})+\frac{\mu-1}{\pi} \int_{\partial K} \frac{l\left(w_{3}\right)(\varphi(t))\left|\varphi^{\prime}(t)\right|}{t(t-\widetilde{\zeta})} d t \\
S_{\partial K} f(z)=\frac{1}{2 \pi i} \int_{\partial K} \frac{f(t)}{(t-z)^{2}} d t, \quad \widetilde{\zeta} \in K, \quad t \in \partial K, \\
l\left(w_{3}\right)(\tau)=\varphi_{2}\left(w_{3}\right)(\tau) /(\mu-1)+\operatorname{Re}\left\{t^{\prime} S d[T f]^{+}(\tau)-\mu_{3} d \alpha^{1} / d S \operatorname{Re} T f(\tau)\right\}, \quad \tau \in \Gamma
\end{gathered}
$$

via $S d\left[\Phi_{l}\right]+(\tau)$ the limit of the function $S d\left[\Phi_{l}\right]+(z)$ is denoted at $z \rightarrow t \in \Gamma$ within the domain $\Omega ; z=\varphi(\zeta)$ - conformal mapping of a single circle $\bar{K}:|\zeta| \leq 1$ on the domain $\Omega ; \zeta=\Psi(z)$ - the function inverse to $z=\varphi(\zeta) ; t^{\prime}=d t / d \sigma, d \sigma-$ the element of a circular arc $\partial K, c_{0}-$ an arbitrary real constant.

We proceed to finding the functions $\Psi_{1}, \Psi_{2}$ from the last two equations in the system (6) that satisfy the conditions (2), (8) on $\Gamma$.

Let's note that the structure of the left-hand sides of the last two equations in (6) is the same as in the case of tangential displacements; they differ only in the right-hand sides. Therefore, for the angles of rotation $\Psi_{1}, \Psi_{2}$ with fixed right-hand sides, we immediately obtain a representation similar to (11):

$$
\psi=\psi_{2}+i \psi_{1}=H_{0}[\tilde{g}(v) ; \tilde{l}(v)]+c_{1}, z \in \Omega,
$$

where the following designations are accepted:

$$
\begin{gathered}
v=v_{2}+v_{1}, \tilde{g}(v)=\left(\tilde{g}_{1}(v)+i \tilde{g}_{2}(v)\right) / 2, \\
v_{j}=w_{3 \alpha^{j}}+\psi_{j}, \widetilde{g}_{j}(v)=k_{0} v_{j}-\beta_{1} L^{j}, \widetilde{l}(v)(t)=\widetilde{\varphi}_{2}(t) /(\mu-1)+h_{2} \widetilde{g}(v)(t), \\
h_{2} \widetilde{g}(v)(t)=-\operatorname{Re}\left\{t^{\prime} S d[T \widetilde{g}(v)]^{+}(t)-\mu_{3} d \alpha^{1} / d s \operatorname{Re} T \widetilde{g}(v)(t)\right\}, \quad t \in \Gamma,
\end{gathered}
$$

$c_{l}$ - arbitrary real constants; the operator $H_{0}[\widetilde{g}(v) ; \tilde{l}(v)]$ is defined by the formula in (13).

In this case, the solvability condition must be satisfied:

$$
\beta_{1}\left(\int_{\Gamma} N^{2}(s) d s+\iint_{\Omega} L^{2} d \alpha^{1} d \alpha^{2}\right)-k_{0} \iint_{\Omega} v_{2} d \alpha^{1} d \alpha^{2}=0,
$$

where $N^{2}, L^{2}$ - the components of the external load, $v_{2} \in W_{p}^{(I)}(\Omega)$ - the function introduced in the formula (15).

Thus, under the conditions (12), (16), the problem A with fixed $w_{3}, v_{j}(j=1.2)$ is solvable with respect to tangential displacements and angles of rotation; its solutions are given by the formulas (13), (14). 
For further research, we use the method of [22]. Taking into account the solution of the system (6) with respect to tangential displacements and angles of rotation, the conditions (2), (7), (8), integral representations (13), (14) are developed for $w_{j}, \Psi_{j}(j=1.2)$. Now let us examine the third equation in (6). Before proceeding to it, we express the deflection $w_{3}$, and its derivatives through $v_{j}(j=1.2)$. Then, using the functions $v=v_{2}+i v_{1}, v_{j}=w_{3 \alpha^{j}}+\Psi_{j}(j=1.2)$, the generalized displacements $w_{j}, \Psi_{j}(j=1.2), w_{3}$, are represented as:

$$
\begin{gathered}
\omega_{0} \equiv \omega_{0}(v)=\omega_{01}(v)+\omega_{02}(v)+\omega_{0}, \Psi \equiv \Psi(v)=\Psi^{0}+\Psi^{I}(v)+\Psi_{*}, \\
w_{3} \equiv w_{3}(v)=w_{30}+w_{30}(v)+w_{3},
\end{gathered}
$$

where

$$
\begin{gathered}
\omega_{0 j}(v)=w_{2 j}(v)+i w_{1 j}(v)=H_{0}\left[f^{j}(v) ; \varphi^{j}(v)\right], j=1.2, \\
\psi^{n}(v)=\psi_{2 n}(v)+i \psi_{1 n}(v)=H_{0}\left[g^{n}(v) ; \tilde{\varphi}^{n}\right], n=0.1, \\
w_{30}=-\int_{(0,0)}^{\left(\alpha^{1}, \alpha^{2}\right)} \psi_{10} d \alpha^{1}+\psi_{20} d \alpha^{2} \\
w_{31}(v)=\int_{(0,0)}^{\left(\alpha^{1}, \alpha^{2}\right)}\left[v_{1}-\psi_{11}(v)\right] d \alpha^{1}+\left[v_{2}-\psi_{21}(v)\right] d \alpha^{2}, \\
\omega_{0^{*}}=-c_{1} k_{4}\left(\alpha^{2}\right)^{2} / 2+\left(k_{4} c_{3}-c_{1}^{2} / 2\right) \alpha^{2}+c_{1} k_{4} / 4+c_{0}, \Psi_{*}(z)=\Psi_{2 *(z)}\left(z \Psi_{1 *(z)}=c_{l},\right. \\
w_{3^{*}}=c_{1} \alpha^{2}+c_{2},
\end{gathered}
$$

$c_{j}, j=\overline{0.3}$ arbitrary real constants.

Further, using (17), the problem $\mathrm{A}$ is reduced to one nonlinear operator equation of the form:

$$
v+G * v=0,
$$

where $G * v$ a nonlinear bounded operator in and for any $v_{j} \in W_{p}^{(I)}(\Omega), j=1.2$, belonging to the ball $\|v\|_{W_{p}^{(1)}(\Omega)}<r$, the estimate is fair $\left\|G_{*} v^{1}-G_{*} v^{2}\right\|_{W_{p}^{(1)}(\Omega)} \leq q_{*}\left\|v^{1}-v^{2}\right\|_{W_{p}^{(1)}(\Omega)}$.

In this case, a solvability condition of the following form appears

$$
\begin{gathered}
\int_{\Gamma}\left(k_{1} \alpha^{1} T^{1}(a)+k_{2} \alpha^{2} P^{2}+P^{3}\right) d s+\iint_{\Omega}\left(k_{1} \alpha^{1} R^{1}+k_{2} \alpha^{2} R^{2}+R^{3}\right) d \alpha^{1} d \alpha^{2}=0, \\
T^{1}(a)=T^{11} d \alpha^{2} / d s-T^{12} d \alpha^{1} / d s,
\end{gathered}
$$

which is performed by choosing the constant $c_{2}$.

\section{Results and discussion}

Let us suppose that the radius $r$ of the ball and the external forces acting on the shell are such that the following conditions are satisfied:

$$
q_{*}<1,\left\|G_{*}(0)\right\|_{W_{p}^{(1)}(\Omega)}<\left(1-q_{*}\right) r .
$$

Then the principle of squeezed mappings can be applied to the equation (19) [26, p. 146], according to which the equation (19) in the ball $\|v\|_{W_{p}^{(1)}(\Omega)}<r$ has a unique solution $v \in$ $W_{p}^{(1)}(\Omega), 2<p<2 /(1-\beta)$.

Thus, the following theorem is true.

Theorem. Let the conditions a), b) from the problem A, and the inequality (20) be satisfied. Then, for the solvability of the geometrically nonlinear equilibrium problem A for shallow elastic shells of the Timoshenko type under boundary conditions (2), it is necessary and sufficient that the condition (12) be satisfied. If it is fulfilled, the problem has a generalized solution $a=\left(w_{1}, w_{2}, w_{3}, \Psi_{1}, \Psi_{2}\right) \in W_{p}^{(I)}(\Omega), 2<p<2 /(1-\beta)$.

\section{Conclusion}

In this paper, the existence theorem is proved within the framework of the shear model by S.P. Timoshenko. When passing to the model by S.P. Timoshenko, the methods previously 
used in the Kirchhoff-Love model cease to work. This is primarily due to the impossibility of constructing energy spaces in which the coercivity inequalities are valid. Therefore, a new analytical method is used, which consists in studying the original system of five equilibrium equations in classical Sobolev spaces under given boundary conditions by reducing it to a single nonlinear operator equation. Analytical (explicit) solutions of shell deformation problem in a geometrically nonlinear formulation are developed. The solvability of boundary value problems describing the equilibrium state of shells in the framework of the simplest Kirchhoff-Love model is quite large. Therefore, consideration of this study provides a significant expansion of engineering application of the theory. Shell structures (various building structures, domes, etc.) require more and more reliable, accurate design data and often pose completely new challenges. Thus, in this paper, an existence theorem is proved and an analytical method is used for finding solutions of geometrically nonlinear, physically linear boundary value problems for elastic shallow isotropic homogeneous shells of the type by S.P. Timoshenko with a different version of hinge-supported edges. The scientific novelty of the considered problem lies in the fact that, first, it is a new, unexplored problem of the mathematical theory of elasticity; secondly, a new method is proposed to solve it that makes it possible to study the solvability of such a class of problems within the framework of more general models for a wider class of elastic structures. The solution of the problem posed makes a contribution to the development of the mathematical theory of elasticity, and can be useful in creating new software packages for elastic structure calculation.

\section{References}

1. R.B. Rikards. Finite element method in the theory of shells and plates. Riga, (1988).

2. V.F. Kirichenko, J. Awrejcewicz, A.F. Kirichenko, A.V. Krysko, V.A. Krysko, International Journal of Non-Linear Mechanics 74, (2015).

3. S.S. Gavryushin, A.S. Nikolaeva. Mechanics of Solids 51, 3 (2016).

4. V.N. Paimushin. Mechanics of Composite Materials 53, 5 (2017).

5. R.A. Kayumov. Mechanics of Solids 52, 5 (2017).

6. V.N. Paimushin, R.A. Kayumov, S.A. Kholmogorov, V.M. Shishkin. Russian Mathematics 62, 6 (2018).

7. V.N. Paimushin, S.A. Kholmogorov, R.A. Kayumov. Uchenye zapiski Kazanskogo universiteta Seriya fiziko-matematicheskie nauki 159, 4 (2017).

8. R.A. Kayumov, B.F. Tazyukov, F.R. Shakirzyanov, I.Z. Mukhamedova. Lobachevskii Journal of Mathematics 40, 3 (2019).

9. R.A. Kayumov, B.F. Tazyukov, I.Z. Mukhamedova. $11^{\text {th }}$ International conference on «Mesh methods for boundary-value problems and applications», Kazan: Ins. of Phys. Publ., (2016).

10. I.I. Vorovich. Mathematical problems of nonlinear theory of shallow shells. Moscow, (1989).

11. N.F. Morozov. Selected two-dimensional problems of elasticity theory. Leningrad, (1978).

12. I.B. Badriev, V.V. Banderov, G.Z. Garipova, M.V. Makarov, R.R. Shagidullin. Applied Mathematical Sciences 9, 82 (2015).

13. I.B. Badriev, M.V. Makarov, V.N. Paimushin. Russian Mathematics 59, 10 (2015).

14. M.M. Karchevskii. Uchenye zapiski Kazanskogo universiteta: Seriya fizikomatematicheskie nauki 158, 3 (2016).

15. M.M. Karchevskii. $11^{\text {th }}$ International conference on «Mesh methods for boundary-value problems and applications». Kazan: Ins. of Phys. Publ., (2016).

16. I.B. Badriev, M.V. Makarov, V.N. Paimushin. Russian Mathematics 61, 1 (2017). 
17. V.N. Paimushin, S.A. Kholmogorov, I.B. Badriev. Lobachevskii Journal of Mathematics 40, 3 (2019).

18. S.N. Timergaliev, A.N. Uglov, L.S. Kharasova. Russian Mathematics 59, 5 (2015).

19. M.G. Ahmadiev, S.N. Timergaliev, L.S. Kharasova. Journal of Siberian Federal University, Mathematics \& Physics 9, 2 (2016).

20. S.N. Timergaliev, L.S. Kharasova. Differential Equations 52, 5 (2016).

21. S.N. Timergaliev. Russian Mathematics 61, 4 (2017).

22. S.N. Timergaliev, L.S. Kharasova. IOP Conf. Series: Materials Science and Engineering 158 (2016).

23. S.N. Timergaliev. Differential Equations 55, 2 (2019).

24. L.S. Kharasova. IOP Conf. Series: Journal of Physics 1158, (2019).

25. K.Z. Galimov. Principles of the nonlinear theory of thin shells. Kazan, (1975).

26. M.A. Krasnosel'skii. Topological methods in the theory of nonlinear integral equations. Moscow, (1956). 